\title{
Milliliter Per Second Per 1.73 Meter Squared
}

National Cancer Institute

\section{Source}

National Cancer Institute. Milliliter Per Second Per 1.73 Meter Squared. NCI Thesaurus.

Code C105505.

A metric unit of volumetric flow rate defined as the rate at which one milliliter of matter travels during the period of time equal to one second per 1.73 meters squared of body surface area. 